\title{
Bacterial etiology and antibiotic susceptibility pattern of female patients with urinary tract infection referred to Imam Khomeini Hospital, Ahvaz, Iran, 2019
}

\author{
Sousan Akrami ${ }^{1.2}$, Reza Abouali², Mohammad Mostafa Olapour², Rozhin Heidary Lal Abady², Hamid \\ Yazdaninejad $^{2}$, Arshid Yousefi-Avarvand $3{ }^{*}$ \\ ${ }^{1}$ Department of Microbiology, Faculty of Medicine, Ahvaz Jundishapur University of Medical Sciences, Ahvaz, Iran \\ ${ }^{2}$ Student Research Committee, Ahvaz Jundishapur University of Medical Sciences, Ahvaz, Iran \\ 3 Department of Medical Laboratory Sciences, School of Para Medicine, Ahvaz Jundishapur University of Medical Sciences, Ahvaz, Iran
}

\begin{abstract}
Urinary tract infection (UTI) is one of the most frequent infections among women, and if untreated could lead to severe complications. The treatment of UTI is difficult due to the appearance of pathogens with increasing resistance to antimicrobial agents. This study thus aimed to determine the bacterial etiological pathogens of UTI and the antibiotic sensitivity pattern of the pathogens isolated. This descriptive cross-sectional study was performed from March to September 2019 on a total of 339 women referred to Imam Khomeini Teaching Hospital in Ahvaz, Iran. Mid-stream urine samples were collected from the patients and were cultured. The presence of significant bacteriuria $\left(>10^{5} \mathrm{CFU} / \mathrm{mL}\right.$ ) was determined using the plate count method. The antimicrobial susceptibility test was done by the standard disk diffusion method. The most frequently isolated pathogens were Escherichia coli (54.8\%), Klebsiella (18.2\%), Pseudomonas aeruginosa (9.9\%), Proteus (8\%), and Acinetobacter (5.1\%). E. coli, as the most common pathogen of UTIs, showed the most resistance to cephalosporins and the least resistance to imipenem. According to the findings, $E$. coli was the most common cause of UTI in our region. Considering the rate of UTI, and the importance of preventing its severe complications, a survey of regional resistance patterns and timely treatment can control the development of its resistant bacteria.
\end{abstract}

Keywords: Urinary tract infection; Antibiotic resistance; Uropathogens

\section{Introduction}

Bacterial infections pose serious threats to public health and affect millions of people annually [1]. Urinary tract infection (UTI), as one of the most common bacterial infections, is known as the second most common cause of infection in the human body $[2,3]$. Thus, the lack of proper diagnosis and treatment of UTIs can lead to severe complications, such as urinary tract disorders, wounds in renal parenchyma,

\footnotetext{
* Corresponding author:

Dr. Arshid Yousefi-Avarvand, Ph.D

Department of Medical Laboratory Sciences, School of Para Medicine, Ahvaz Jundishapur University of Medical Sciences, Ahvaz, Iran

Tel/Fax: +986133738317

Email: arshid.yousefi5@gmail.com

http://orcid.org/o0oo-0002-3987-9820
}

Received: July, 26, 2020

Accepted: August, 09, 2020 hypertension, and uremia. In pregnant women, UTI can also lead to preterm labor and even abortion $[4,5]$. The urinary tract is naturally devoid of any microorganisms, and infection occurs when any bacteria, virus, fungus, or parasite infects the urinary tract [2]. The rate of UTIs in developing countries is estimated to be at least 250 million cases per year. This infection is usually caused by bacteria, especially Gram-negative bacteria, such as Escherichia coli 
which is seen in all age groups in both sexes, though it is more likely to occur in young women $[6,7]$.

Over the half of women develop UTI at least once in their lifetime, primarily in the lower urinary tract, which includes the urethra and bladder. Therefore, identifying the type of bacteria that cause UTI and prescribing the appropriate antibiotics to prevent its main complications such as urinary tract disorders, premature birth, and abortion seem indispensable [8]. Antimicrobial sensitivity testing lab reports, intended to determine the bacterial etiological pathogens of UTI, are usually released within 48 hours after receiving the samples in the laboratory. For this reason, in most cases, experimental treatment is recommended by the physicians, as antibiotic resistance pattern studies of pathogens and epidemiologic studies can be helpful in the treatment of UTIs [9]. In addition, as the resistance pattern in any infectious area in the body changes over time, new studies are thus needed to determine the etiological pathogens of the infection. Given the prevalence of UTIs and the importance of preventing its severe complications, this study was thus intended to determine the most common strains causing UTIs and the antibiotic resistance pattern of such strains in the city of Ahvaz.

\section{Materials and Methods}

2.1. Urine Samples

The present study was performed on 341 patients admitted to Imam Khomeini Teaching Hospital in Ahvaz, Iran, during a six-month period from April to September 2019. To that end, the samples collected from midstream urine of the patients were transferred to the laboratory. Then, they were cultured in blood agar and MacConkey agar medium (Merck, Germany) and were incubated at $37^{\circ} \mathrm{C}$ for 24 hours. Cultures containing more than one bacterial strain were considered as contamination and excluded. All the isolates were identified using the conventional bacteriologic methods (i.e., microscopical and appropriate biochemical tests).

2.2. Antibiotic susceptibility

Antimicrobial sensitivity tests were carried out by the disc diffusion technique using Muller Hinton agar (Merck, Germany) in accordance with the Clinical and Laboratory Standards Institute (CLSI) recommendations [10]. Imipenem (10 $\mu \mathrm{g})$, Ampicillin (10 $\mu \mathrm{g})$, Amikacin (30 $\mu \mathrm{g})$, Cefazolin (30 $\mu \mathrm{g})$, Ceftriaxone (30 $\mu$ g), Cephalexin (30 $\mu \mathrm{g})$, Cefixime (5 $\mu \mathrm{g})$, Gentamicin (10 $\mu \mathrm{g})$, Vancomycin (30 $\mu \mathrm{g}$ ) and Penicillin (10 units) all from PadtanTeb, Iran.

\section{Results}

Totally, 352 bacterial strains were isolated from the 339 urine samples of women screened for bacterial UTIs in this study. Data analysis showed that the highest prevalence was for E. coli (54.8\%), while Enterococcus faecalis (1.1\%) was the least abundant (Table 1). Table 2 shows the resistance pattern of isolates to different antibiotics. The results of antibiotic susceptibility test of the bacterial isolates indicated that a high proportion of the test organisms were sensitive to amikacin and imipenem. Amikacin was, in turn, found to be very effective against all the isolates. Besides, most of the isolates were sensitive to imipenem, and high-level resistance was seen in ceftriaxone, cephalexin, and cefazolin.

Table 1. Distribution of organisms isolated from female patients with UTIs

\begin{tabular}{lc}
\hline Organism & Number (\%) \\
\hline Escherichia coli & $193(54.8)$ \\
\hline Klebsiella pneumoniae & $64(18.2)$ \\
\hline Pseudomonas aeruginosa & $35(9.9)$ \\
\hline Proteus spp. & $28(8)$ \\
\hline Acinetobacter spp. & $18(5.1)$ \\
\hline Staphylococcus aureus & $10(2.8)$ \\
\hline Enterococcus faecalis & $4(1.1)$ \\
\hline
\end{tabular}




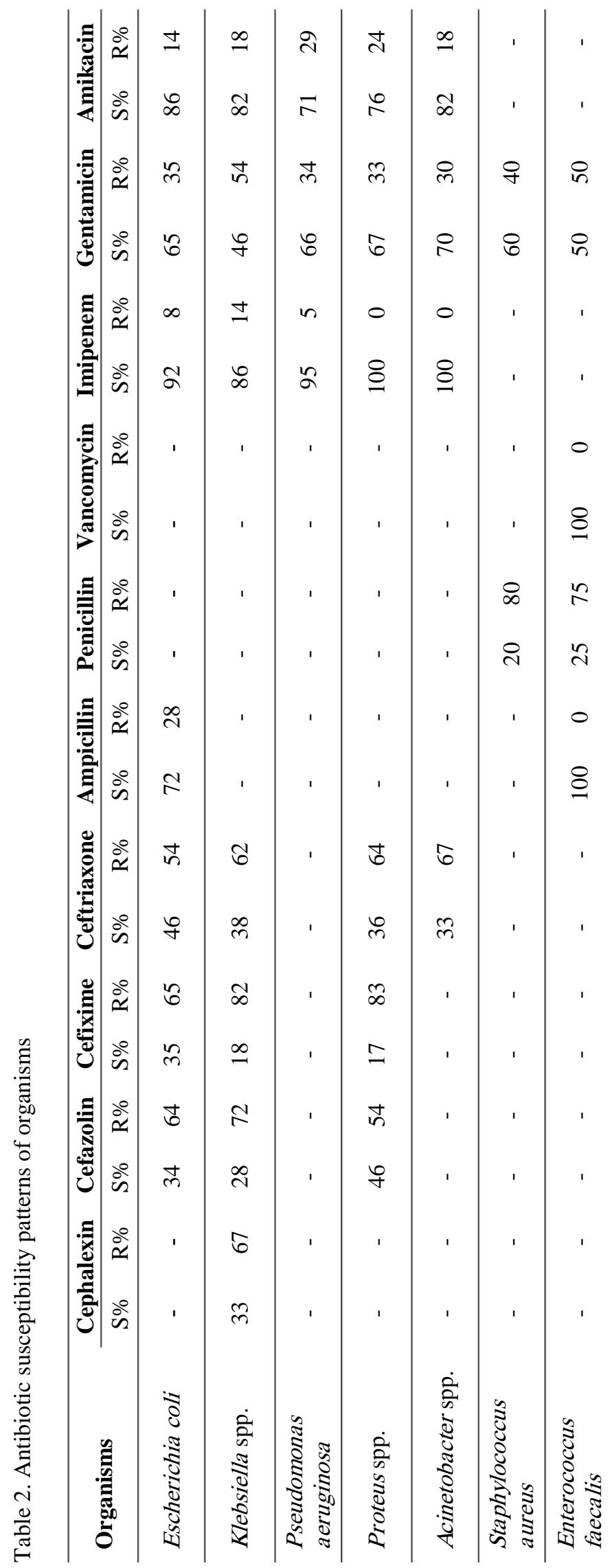

\section{Discussion}

The arbitrary use of antibiotics in many cases causes UTI not to be isolated, which in turn may lead to the failure of treatment [11]. To determine the antibiotic resistance pattern of isolates from a urine culture, there are several reports in different parts of the world, including Iran. For instance, Raeeszadeh et al. studied 114 patients with urinary duct infections and shows that the most commonly isolated pathogen was E. coli (74\%), while the least isolated one was related to Pseudomonas spp. (1\%). Also, the most and the least susceptibility in $E$. coli were for nitrofurantoin and sulfamethoxazole, respectively [12]. In another research, Jabrodini et al. studied 1300 urine samples to determine the etiological pathogens causing UTI and their antibiotic resistance profile. Their results showed that 247 samples (19\%) were positive for bacterial growth, 148 urine samples (11.3\%) were positive for $E$. coli and Klebsiella pneumoniae. Besides, the most and the least antibiotic resistance in $E$. coli was found to be to nalidixic acid (59.3\%) and nitrofurantoin (22.2\%), respectively. For $K$. pneumoniae strains, the most and the least antibiotic resistance was observed in nitrofurantoin (67.5\%) and ciprofloxacin (25\%) [13]. Similarly, studying 462 urine samples collected from patients with UTI in Bangladesh, reported that 100 of the samples were found to be culture positive. Indeed, E. coli (70\%) was predominant, followed by Klebsiella spp. (11\%) and Enterococcus spp. (6\%). The results also revealed that about $96 \%$ of uropathogens were sensitive against imipenem, and $75 \%$ against amikacin. Moreover, the most antibiotic resistance in $E$. coli was to nalidixic acid (84.3\%) [14]. In other research, Shokoohi et al. studied 1400 urine samples from outpatients in Hamadan, 235 samples were positive, of which 105 cases $(44.7 \%)$ were related to men, and 130 cases (55.4\%) were women. E. coli isolated from 141 (60\%) cases and was the most common organism. In this study, the resistance of $E$. coli to ceftriaxone and cefixime was $55 \%$ and 50\%, respectively. The study showed that the most effective antibiotic for $E$. coli is amikacin (70\% sensitivity) [15].

As main limitations of retrospective studies, we only access to the results of locally available antibiotics. Also, the results obtained from a regional hospital and may not be generalized to entire area.

Overall, $E$. coli isolates showed high resistance to conventional antibiotics for the treatment of UTIs. In 
our study, the best antibiotics to treat $E$. coli UTIs were found to be imipenem and amikacin. The findings also showed that the level of antibiotic resistance in different regions in human body depends on different factors, including antibiotic use patterns. For this reason, periodic monitoring of antibiotic resistance is recommended to control the infection at its source.

\section{Author Contributions}

Conception or design of the work AY, SA; Data collection: RA, RH; Data analysis and interpretation MMO, HY, RA; Drafting the article: SA; Critical revision of the article: AY. All authors read and approved the final version of manuscript.

\section{Conflict of Interests}

Authors declare there is no conflict of interest.

\section{Ethical declarations}

This article does not contain any studies with human participants and only samples leftovers in laboratory were used. The study design was approved by the regional Ethics Committee of Ahvaz Jundishapur University of Medical Sciences (IR.AJUMS.REC.1398.837).

\section{Financial Support}

This work was supported by a grant number 98s81 from Vice Chancellor for Research, Ahvaz Jundishapur University of Medical Sciences.

\section{References}

1. Bloom DE, Cadarette D. Infectious Disease Threats in the Twenty-First Century: Strengthening the Global Response. Front Immunol. 2019; 10:549.

2. Flores-Mireles AL, Walker JN, Caparon M, Hultgren SJ. Urinary tract infections: epidemiology, mechanisms of infection and treatment options. Nat Rev Microbiol. 2015; 13(5):269-84.

3. Najar MS, Saldanha CL, Banday KA. Approach to urinary tract infections. Indian J Nephrol. 2009; 19(4):129-39.

4. Beahm NP, Nicolle LE, Bursey A, Smyth DJ, Tsuyuki RT. The assessment and management of urinary tract infections in adults:
Guidelines for pharmacists. Can Pharm J (Ott). 2017; 15O(5):298305 .

5. Loh K, Sivalingam N. Urinary tract infections in pregnancy. Malays Fam Physician. 2007; 2(2):54-7.

6. Beyene G, Tsegaye W. Bacterial uropathogens in urinary tract infection and antibiotic susceptibility pattern in jimma university specialized hospital, southwest ethiopia. Ethiop J Health Sci. 2011; 21(2):141-6.

7. Tandogdu Z, Wagenlehner FM. Global epidemiology of urinary tract infections. Curr Opin Infect Dis. 2016; 29(1):73-9.

8. Minardi D, d'Anzeo G, Cantoro D, Conti A, Muzzonigro G. Urinary tract infections in women: etiology and treatment options. Int J Gen Med. 2011; 4:333-43.

9. Davenport M, Mach KE, Shortliffe LMD, Banaei N, Wang TH, Liao JC. New and developing diagnostic technologies for urinary tract infections. Nat Rev Urol. 2017; 14(5):296-310.

10. CLSI. Performance Standards for Antimicrobial Susceptibility Testing; 30th ed. CLSI supplement M10o. Wayne, PA: Clinical and Laboratory Standards Institute; 2020.

11. Akram M, Shahid M, Khan AU. Etiology and antibiotic resistance patterns of community-acquired urinary tract infections in J N M C Hospital Aligarh, India. Ann Clin Microbiol Antimicrob. 2007; 6:4.

12. Raeeszadeh M, Ahmadi E, Shafiee M. Identification of the antibiotic resistance patterns in bacteria isolated from urinary tract infections in patients admitted to Shahid Ghazi Hospital-Sanandaj in the first 6 months of 1393. Razi J Med Sci. 2016; 23(147):11-7. 13. Jabrodini A, Heidari F, Taghavi SF, Shokouh MR. The Investigation of Frequency and Antibiotic Resistance Pattern of Escherichia coli and Klebsiella pneumoniae Isolated From Urinary Tract Infection in Outpatients Referred to Amiralmomenin Ali Hospital in Gerash City in 2017: A Short Report. J Rafsanjan Univ Med Sci. 2018; 17(1):75-84.

14. Noor A, Shams F, Munshi S, Hassan M, Noor R. Prevalenceand antibiogram profile of uropathogens isolated from hospital and community patients with urinary tract infections in Dhaka City. $\mathrm{J}$ Bangladesh Acad Sci. 2013; 37(1):57-63.

15. Shokoohi R, Samarghandi M, Dargahi A, Alikhani MY, Roshanaei G, Moradi Golrokhi M. Investigation of Antibiotic Resistance Pattern of Bacteria Causing the Urinary Tract Infection in Urine Samples of Patients Admitted in and Referred to Shahid Beheshti Hospital in Hamadan. Pajouhan Sci J. 2019; 17(3):34-40. 